ARTICLE

\title{
Ultra-fast self-assembly and stabilization of reactive nanoparticles in reduced graphene oxide films
}

Yanan Chen ${ }^{1, \star}$, Garth C. Egan ${ }^{2,3, \dagger, \star}$, Jiayu Wan ${ }^{1, \star}$, Shuze Zhu ${ }^{4}$, Rohit Jiji Jacob ${ }^{2,3}$, Wenbo Zhou ${ }^{2,3}$, Jiaqi Dai ${ }^{1}$, Yanbin Wang ${ }^{1}$, Valencia A. Danner ${ }^{1}$, Yonggang Yao ${ }^{1}$, Kun Fu¹, Yibo Wang ${ }^{1}$, Wenzhong Bao ${ }^{1}$, Teng $\mathrm{Li}^{4}$, Michael R. Zachariah ${ }^{2,3}$ \& Liangbing $\mathrm{Hu}^{1}$

Nanoparticles hosted in conductive matrices are ubiquitous in electrochemical energy storage, catalysis and energetic devices. However, agglomeration and surface oxidation remain as two major challenges towards their ultimate utility, especially for highly reactive materials. Here we report uniformly distributed nanoparticles with diameters around $10 \mathrm{~nm}$ can be self-assembled within a reduced graphene oxide matrix in $10 \mathrm{~ms}$. Microsized particles in reduced graphene oxide are Joule heated to high temperature $(\sim 1,700 \mathrm{~K})$ and rapidly quenched to preserve the resultant nano-architecture. A possible formation mechanism is that microsized particles melt under high temperature, are separated by defects in reduced graphene oxide and self-assemble into nanoparticles on cooling. The ultra-fast manufacturing approach can be applied to a wide range of materials, including aluminium, silicon, tin and so on. One unique application of this technique is the stabilization of aluminium nanoparticles in reduced graphene oxide film, which we demonstrate to have excellent performance as a switchable energetic material.

\footnotetext{
${ }^{1}$ Department of Materials Science and Engineering, University of Maryland College Park, 1208 Engineering Lab Building, College Park, Maryland 20742, USA.

${ }^{2}$ Department of Chemical and Biomolecular Engineering, University of Maryland College Park, College Park, Maryland 20742, USA. ${ }^{3}$ Department of Chemistry and Biochemistry, University of Maryland College Park, College Park, Maryland 20742, USA. ${ }^{4}$ Department of Mechanical Engineering, University of Maryland College Park, College Park, Maryland 20742, USA. † Present address: Materials Science Division, Lawrence Livermore National Laboratory, Livermore, California 94550, USA. * These authors contributed equally to this work. Correspondence and requests for materials should be addressed to M.R.Z. (email: mrz@umd.edu) or to L.H. (email: binghu@umd.edu).
} 
$\mathrm{F}$ rom the Lycurgus cup as early as the fourth century to modern cancer sensing and energy storage, nanoparticles are widely used in physical ${ }^{1-3}$, chemical $^{4-6}$, environmental $^{7,8}$, biological ${ }^{9,10}$ and medical applications ${ }^{11,12}$, owing to their interesting size-dependent properties. Numerous scalable and low-cost methods for making high-quality nanoparticles have been reported and many types of nanoparticles are commercially available ${ }^{13,14}$. These synthesis methods can be generally classified into two categories. The first category features 'top-down' approaches, usually solid-phase methods, including ball milling ${ }^{15,16}$, pulsed laser ablative deposition $^{17-19}$, exploding wire ${ }^{13,20,21}$ and so on. The second category features 'bottom-up' approaches, such as chemical and physical vapour deposition 22,23 and liquid-phase chemical synthesis ${ }^{24-33}$. Although many of these approaches have been quite successful, there are significant challenges that still exist. This is especially true for non-noble metals that can be highly reactive and tend to suffer from surface oxidation and agglomeration, which can significantly constrain nanoparticle functionality ${ }^{34-36}$. A fast, low-cost and scalable approach to manufacturing uniformly distributed nanoparticles, especially for energetic nanoparticles, without these impairments is urgently needed. At the same time, nanoparticles hosted in a conductive matrice are ubiquitous in electrochemical energy storage, catalysis and energetic devices, where a conductive matrix can provide fast electron transport for the operations of the devices. Usually, nanoparticles are synthesized first and then assembled into a conductive carbon matrix ${ }^{37}$. For reactive nanoparticles, significant oxidation often occurs during the two steps, which presents a major challenge to the effective use of these nanoparticles ${ }^{38}$.

Here we report an ultra-fast (as fast as $2 \mathrm{~ms}$ ) process to produce uniformly distributed nanoparticles in a conductive reduced graphene oxide (RGO) matrix by directly Joule heating a metal/ semiconductor-RGO film (Fig. 1a) to a high temperature $(\sim 1,700 \mathrm{~K}$ or higher). Microsized metal or semiconductor particles used as precursors are melted under high-temperature Joule heating, then self-assemble into nanoparticles on cooling. Agglomeration and coalescence of the nanoparticles, which is driven by surface energy minimization, is suppressed by the defects of RGO in-plane and RGO layers out-of-plane (Fig. 1b). These defects serve as barriers for atom migration, to keep the uniformly distributed nanoparticles separated. The particles within the matrix during heating are protected from oxidation by the RGO sheets, owing to the impermeability of RGO to $\mathrm{O}_{2}$ and $\mathrm{H}_{2} \mathrm{O}^{39}$. Thus, RGO sheets serve as a perfect host material for such unique high-temperature process, owing to their defect sites and high melting temperature (stable up to $3,300 \mathrm{~K})^{40-45}$. Furthermore, this high-temperature process for in situ synthesis of nanoparticles without agglomeration and surface oxidation is applicable to any materials with a lower melting point than $3,300 \mathrm{~K}$. In this study, we demonstrate fast nanoparticle formation for aluminium $(\mathrm{Al})$, silicon $(\mathrm{Si})$, tin $(\mathrm{Sn})$, gold $(\mathrm{Au})$ and palladium $(\mathrm{Pd})$. These materials in their nanoparticle form have been widely used in energetics, energy storage, optical, sensing and catalytic applications. We choose nAl-RGO to demonstrate its superior properties as an energetic material. Furthermore, we combined three-dimensional (3D) printing with this technique for both precise shape control and scalable manufacturing of nanoparticles-RGO architectures (Fig. 1c).

\section{Results}

Synthesis of nanoparticles in RGO network. The nanoparticleRGO films were fabricated with a homemade setup, where in situ monitoring of the temperature and electrical conductivity during Joule heating can be achieved (Fig. 2a). To obtain the nanoparticle-RGO structure, we started with microsized particles embedded within an RGO thin film prepared by vacuum filtration (Supplementary Fig. 1 and Supplementary Methods). The films were fixed on a glass holder with current applied to achieve high temperature in a vacuum chamber (Supplementary Fig. 2). A typical freestanding Al-RGO film before and during Joule heating is shown in Fig. 2b. The Joule heating process to high temperatures further reduced the $\mathrm{RGO}^{46-48}$, leading to a large conductivity increase in the Al-RGO film. Two detailed voltage sweeps of the first and second cycle of the film are shown in Fig. 2c. In the first heating process, the conductivity of Al-RGO film increases more than 100 times (from 1.3 to $139.1 \mathrm{~S} \mathrm{~cm}^{-1}$ ) on Joule heating. After cooling down to room temperature (Fig. 2d), the conductance of the film was $75 \mathrm{~S} \mathrm{~cm}^{-1}$. In the hightemperature treatment process, a sequence of photo images with increasing applied electrical power is shown in Fig. 2e. To monitor the temperature of the Al-RGO sample, its emitted radiation was recorded in situ by an optical fibre and spectrometer. The emission spectrum (Fig. 2f) of the Al-RGO device was then fitted to the Blackbody radiation equation, to extract the temperature of the film (Supplementary Fig. 3 and Supplementary Methods). In the Al-RGO experiment, we obtained different temperatures of 1,380, 1,530, 1,710, 1,780 and $2,200 \mathrm{~K}$ at $50,75,100,125$ and $150 \mathrm{~mA}$, respectively (Fig. $2 \mathrm{~g}$ ). To demonstrate structural changes, Raman spectra of the Al-RGO films before and after 2,200 K treatments were obtained (Fig. 2h). We observed a largely increased intensity ratio of $\mathrm{D}\left(1,350 \mathrm{~cm}^{-1}\right)$ a

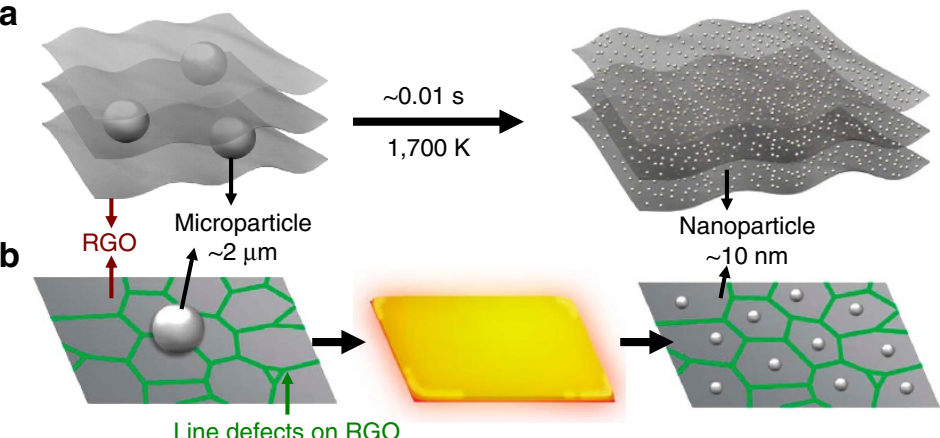

C

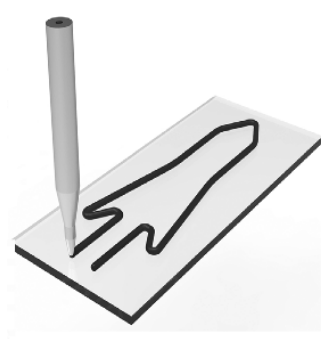

Figure 1 | Schematic of in situ nanoparticles self-assembly process. (a) Microsized particles in a conductive RGO network matrix (left) are self-assembled into nanoparticles (right) driven by direct Joule heating for $\sim 10 \mathrm{~ms}$. (b) Proposed mechanism for nanoparticle formation, where a microsized particle melts on heating and self-assembles into nanoparticles due to confinement by the defects of the RGO sheet. (c) The raw materials are 3D printable, where nanoparticles can be formed in a conductive RGO matrix. 


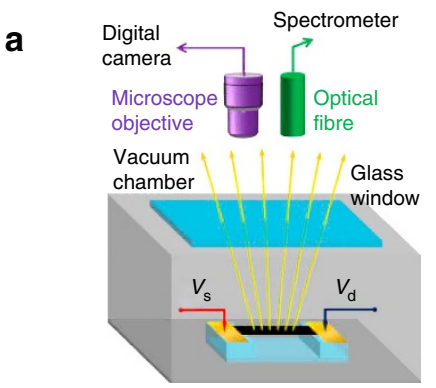

e

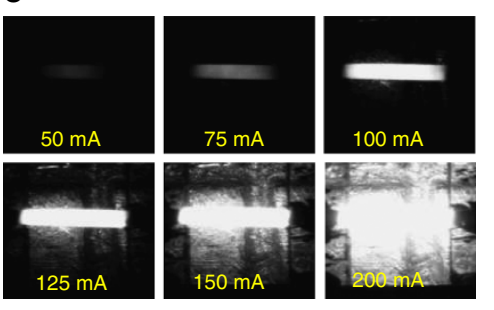

b

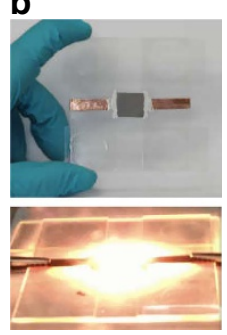

C
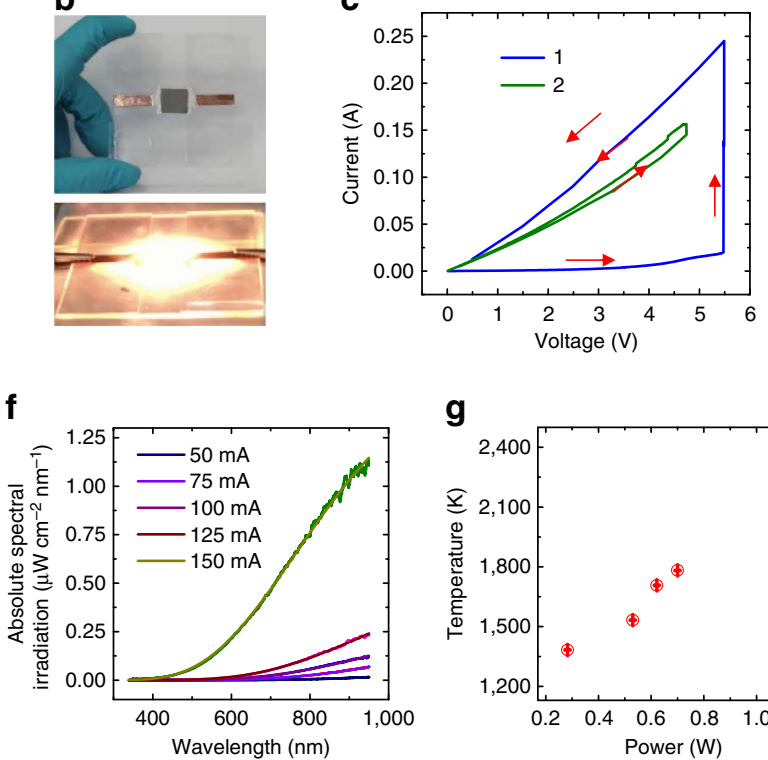

g

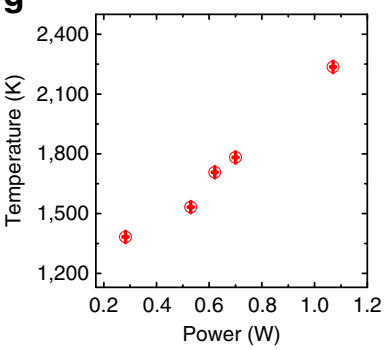

d

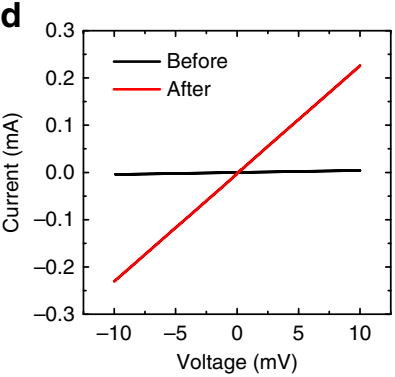

h

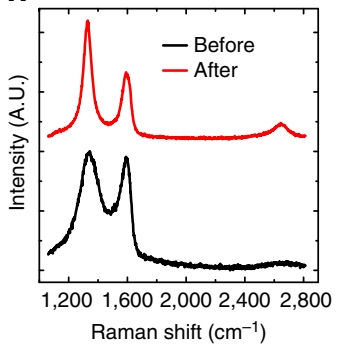

Figure 2 | Characterizations of fast nanoparticle formation process. (a) Home-made optical measurement setup for metal-RGO high-temperature

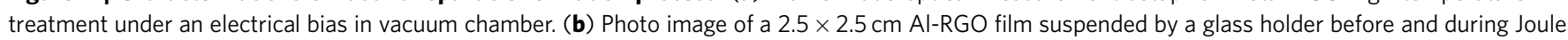
heating treatment. (c) I-V curve of an AI-RGO film (width: $506 \mu \mathrm{m}$, length: $636 \mu \mathrm{m}$ ) during a high-temperature treatment process. (d) I-V curve of Al-RGO film before and after treatment. (e) A sequence of photo images to show the Al-RGO film (width: $380 \mu \mathrm{m}$, length: $1917 \mu \mathrm{m}$ ) under Joule heating with an increasing applied electrical power. (f) Light emission spectra of Al-RGO film at different current densities. (g) Temperature of Al-RGO film at different power. (h) Raman spectroscopy of Al-RGO film before and after high-temperature treatment.

to $\mathrm{G}$ peak $\left(1,600 \mathrm{~cm}^{-1}\right)\left(I_{\mathrm{D}} / I_{\mathrm{G}}\right)$, from 1.03 to 1.83 , after Joule heating. The Al-RGO film maintains a high $I_{\mathrm{D}} / I_{\mathrm{G}}$ ratio in the high-temperature treatment process (Supplementary Fig. 4 and Supplementary Note 1), which is strikingly different with that of pure RGO films (Supplementary Fig. 5). The difference in Raman spectrum is possibly correlated with the formation of $\mathrm{Al}$ nanoparticles. As we believe the nanoparticles are stabilized by the defect sites, the defect density is thus stabilized by the nanoparticles such that the defect density is constant in Al-RGO ${ }^{49}$, whereas the defects in pure RGO film are self-healed at high temperature $\mathrm{e}^{50}$.

Characterization of nanoparticles in RGO network. To understand the formation of nanoparticles during hightemperature treatment, scanning electron microscopy (SEM) and transmission electron microscopy (TEM) are applied to characterize the Al-RGO before and after Joule heating treatment at $2,200 \mathrm{~K}$ for $1 \mathrm{~min}$. The $\mathrm{Al}$ particles in RGO film before treatment have an average diameter of $2 \mu \mathrm{m}$ (Fig. 3a). After treatment, high-density $\mathrm{Al}$ nanoparticles were dispersed uniformly in RGO layers (Fig. 3b,c), with sizes ranging from 1.0 to $25.0 \mathrm{~nm}$ (Fig. 3c, inset). The interior structure (Supplementary Fig. 6) and the cross-sectional morphology of Al-RGO film (Supplementary Fig. 7) confirm high-density distribution of $\mathrm{Al}$ nanoparticles within the entire RGO network. The rings of distinct spots in the selected area diffraction pattern indicate polycrystalline structure (Fig. 3d-f), which results from multiple nanoparticles in the electron diffraction area. The spacing of these rings is consistent with face-centered cubic (FCC) Al. The brighter, continuous rings agree with graphite. It is noteworthy that this particular sample was exposed to air before TEM analysis; however, from the diffraction pattern, we did not observe the formation of $\mathrm{Al}_{2} \mathrm{O}_{3}$. This result indicates that the $\mathrm{RGO}$ can serve as an oxidation barrier for zero-valent metals. We also demonstrated that the proposed heating method for metal nanoparticles synthesis can be applied to a broad range of materials, such as $\mathrm{Pd}, \mathrm{Au}, \mathrm{Si}$ and $\mathrm{Sn}$ (Fig. 3g-j and Supplementary Methods).

Ultra-fast synthesis of Al nanoparticles. The longer a material is heated, the more significant heat losses become. Therefore, to make this process as energy efficient as possible, it is important to quantify the minimal time needed to achieve nanoparticle synthesis. To this end, we quantified the high-temperature selfassembly of $\mathrm{Al}$ nanoparticles in RGO matrix driven by current applied for only $2 \mathrm{~ms}$ (Supplementary Figs 8 and 9). The fast current pulse through the Al-RGO sample $(1,730 \mu \mathrm{m}$ long, $960 \mu \mathrm{m}$ wide and $4 \mu \mathrm{m}$ thick) was programed to go from 0 to $1.05 \mathrm{~A}$ within $1 \mathrm{~ms}$ (Fig. 4a). The materials response was monitored with a specially designed sub-millisecond diagnostics (Supplementary Fig. 10 and Supplementary Note 2). The output spectra from the 24 channels plotted versus time with the wavelength axis shows the central wavelength of each channel (Fig. 4b). The intensity peaks occur within the millisecond time frame. The time-resolved spectrum was used to extract the temperature (Fig. 4c) accompanied by the intensity profile on the $800 \mathrm{~nm}$ channel. The temperature rise time and cooling time were estimated to be $\sim 900 \mu \mathrm{s}$ and $\sim 9 \mathrm{~ms}$, respectively, with the cooling occurring after Joule heating via conduction to the holder and radiation to the surroundings (no convection due to the experiments being done under vacuum). As the photo multiplier tube (PMT) operated in the visible range, the lowest temperatures it could detect with reasonable accuracy was around $1,000 \mathrm{~K}$ and, as the intensity levels dropped with the sample cooling down, the signal-to-noise ratio was substantially reduced resulting in inaccurate temperature prediction at the later stages. The synthesis process of $\mathrm{Al}$ nanoparticles is around $10 \mathrm{~ms}$, which is much faster than many traditional synthesis methods for nanoparticles, such as ball milling, physical vapour deposition and liquid-phase chemical synthesis. The highest temperature achieved for this nAl-RGO sample was $1,730 \mathrm{~K}$ in the ultra-fast heat process. After 
a

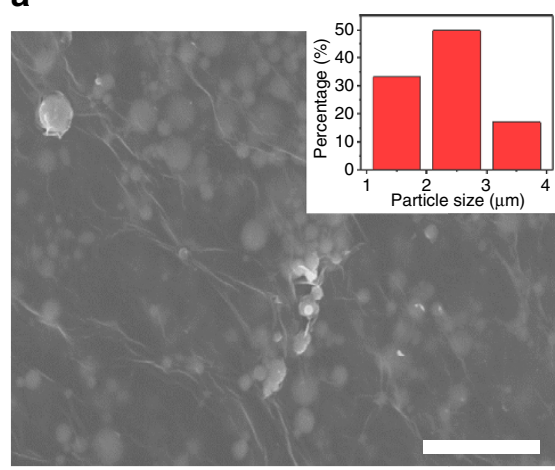

d

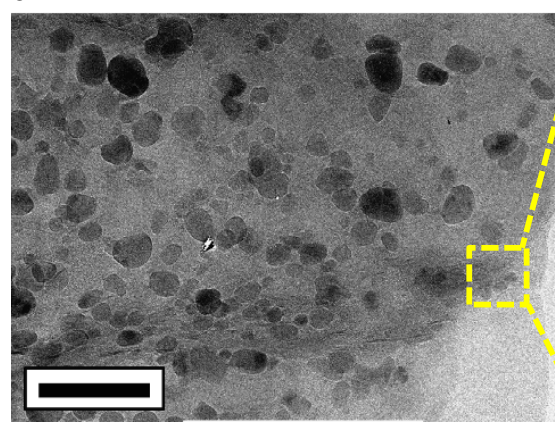

g

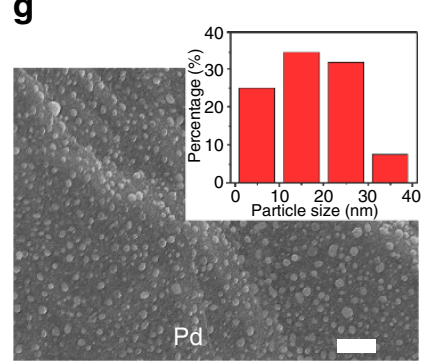

b

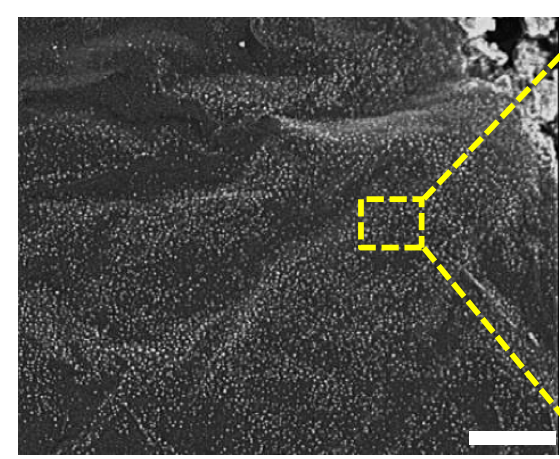

e

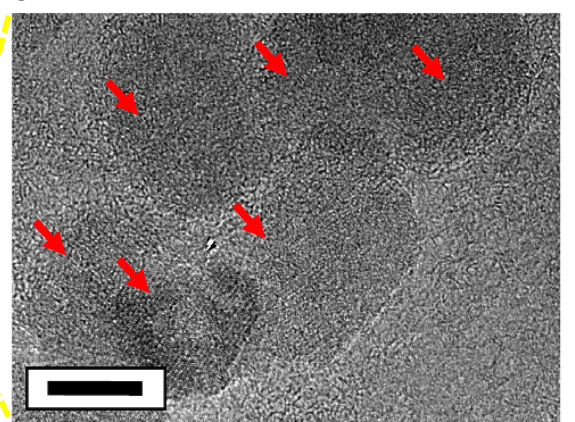

C

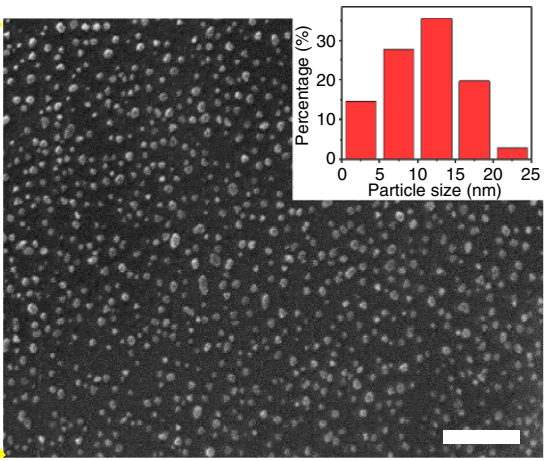

f

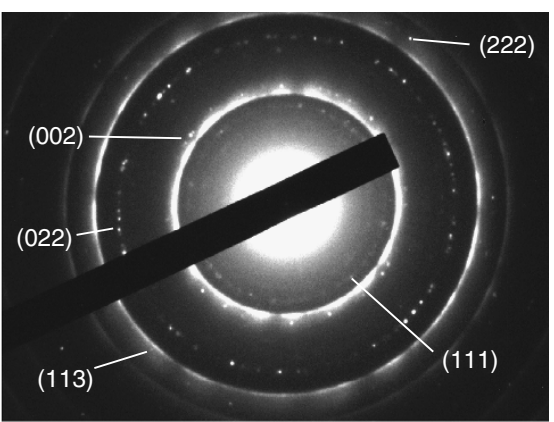

j

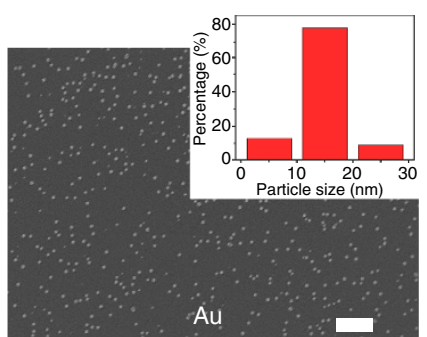

i

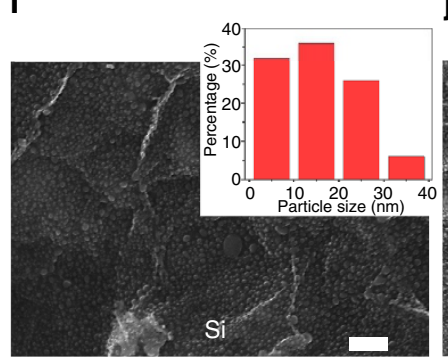

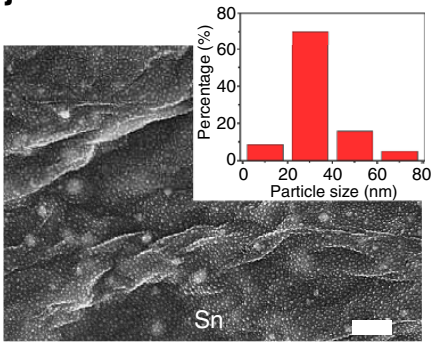

Figure 3 | Characterization of nanoparticles formed in RGO network. (a) SEM image of RGO films with embedded Al microsized particles. (b,c) SEM images of Al nanoparticles after 2,200 K Joule heating for $1 \mathrm{~min}$. (d-f) TEM images of nAl-RGO with different magnifications and the corresponding selected area diffraction pattern patterns. (g-j) SEM images of nanoparticles formed in RGO network with Pd, Au, Si and Sn. Particle size distribution are also shown in the insets. Scale bar, (a) $10 \mu \mathrm{m},(\mathbf{b}) 1 \mu \mathrm{m},(\mathbf{c}) 200 \mathrm{~nm},(\mathbf{d}) 100 \mathrm{~nm},(\mathbf{e}) 10 \mathrm{~nm},(\mathbf{g}, \mathbf{h}) 200 \mathrm{~nm},(\mathbf{i}) 300 \mathrm{~nm}$ and (j) $1 \mu \mathrm{m}$.

cooling down, the obtained $\mathrm{Al}$ nanoparticles have an average size of $10 \mathrm{~nm}$. A typical morphology is shown in Fig. 4d.

Formation mechanism of nanoparticles in RGO. We propose the following mechanism for the ultra-fast, in situ nanoparticles formation at high temperatures. Al microsized particles melt under Joule heating and presumably disperse throughout the films. As cooling takes place, the nucleation occurs around the defects on the RGO network and accretes $\mathrm{Al}$ atoms to form ultra-fine nanoparticles. We have noticed that the temperature involved in our heating may be beyond the bulk boiling point of aluminium. To this regard, we cannot exclude the possibility of aluminium vapourization in the high-temperature treatment process. However, owing to the encapsulation effect of RGO layers, even if there are vapourized aluminium atoms, they will remain in between RGO layers. Next, during the cooling process, these vapourized aluminium atoms can re-group and condense into new nanoparticles. Therefore, such mechanism is in-effect similar to melting, but with an intermediate vapourizing and condensation stage, before reshaping into new nanoparticles. This is an interesting topic to investigate in future studies. A striking feature of the resulting nanoparticles is their superior structural stability. One ubiquitous challenge to nanoparticles is their tendency to merge and agglomerate at high temperature, driven by surface energy minimization. However, the nanoparticles formed at $2,200 \mathrm{~K}$ in our experiments remain stable without appreciable merging or agglomeration. We attribute the superior stability of the resulting nanoparticles to a barrier effect of the defects in RGO, as to be shown by atomistic modelling analysis. Defects (for example, vacancies, grain boundaries and voids) inevitably form during the synthesis of RGO. We investigate the effect of defects in RGO on the dispersion of nanoparticles and find that the defects can serve as an effective barrier so that the migration of nanoparticles is constrained to be within the domain demarcated by neighbouring defects. As a result, nanoparticles remain isolated from each other and thus no agglomeration and merging occur.

We first demonstrate the barrier effect of the defects (for example, an open slit) on a single foreign atom (for example, an $\mathrm{Al}$ atom) through a theoretical model (Fig. 5a, Supplementary Fig. 11 and Supplementary Note 3). As shown in the right panel of Fig. 5a, the potential energy of the system reaches its minimum as the $\mathrm{Al}$ atom is far away from the defect. As the $\mathrm{Al}$ atom 
a
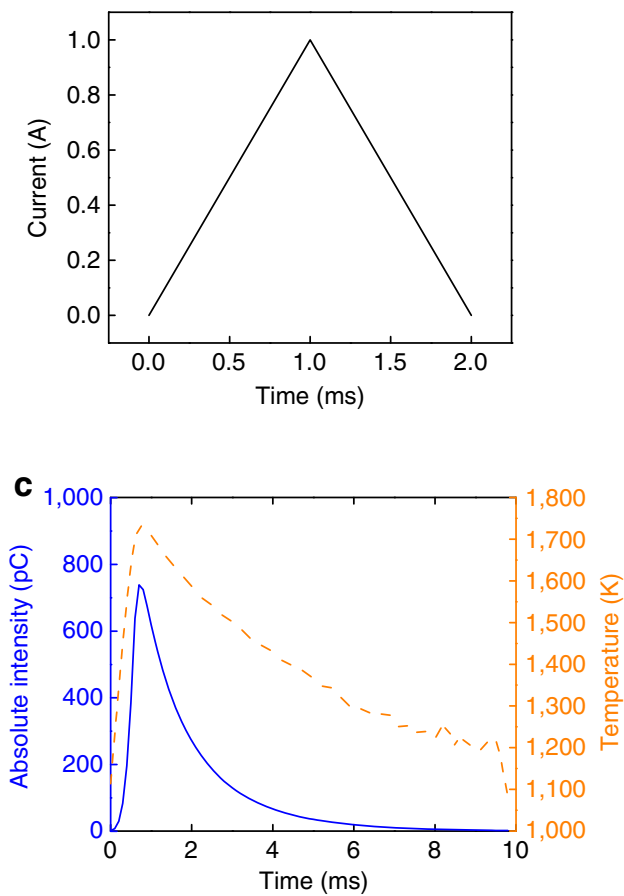

b

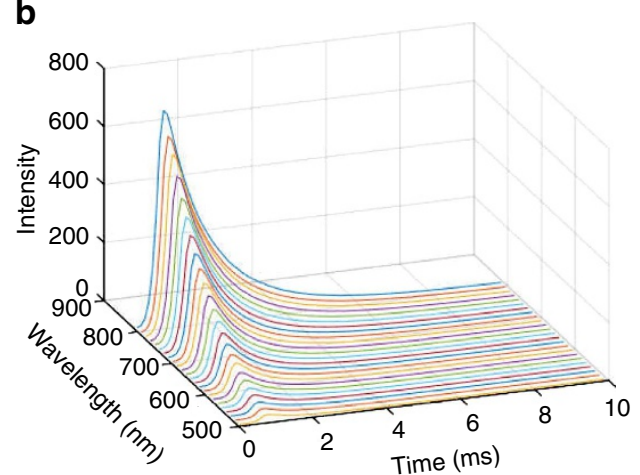

d

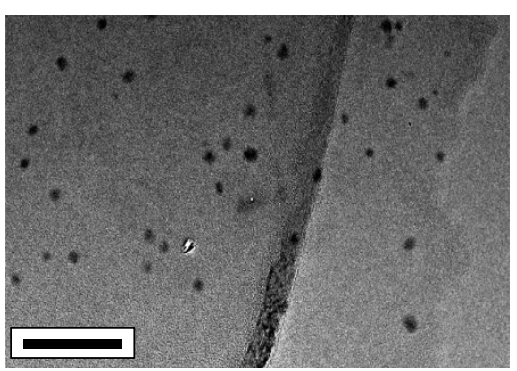

Figure 4 | Characterization of ultra-fast Al nanoparticles formation process. (a) Current value programmed for the high-speed spectroscopic temperature measurement for AI-RGO sample. (b) Emission spectra of AI-RGO film in the ultra-fast heating process. (c) Temperature and intensity profile on the $800 \mathrm{~nm}$ channel in the ultra-fast heating process. (d) TEM image of Al nanoparticles formed in RGO network after ultra-fast heating. Scale bar, $50 \mathrm{~nm}(\mathbf{d})$.

approaches the slit defect, the total potential energy of the system increases, peaks near the edge of the slit defect and then decreases slightly as it approaches the centre of slit defect width. In other words, there exists an energy barrier for the $\mathrm{Al}$ atom to migrate across the slit defect and a local minimum that could pin the atom at the defect. The energy barrier increases as the width of the slit defect increases. As such an energy barrier essentially results from the change of atom density at the defect site ${ }^{51}$ (that is, missing atoms from a perfect material lattice structure), the barrier effect of defects is generally applicable to various defect types and foreign atom species ${ }^{51}$. We next perform molecular dynamics (MD) simulations, to demonstrate that such a defectinduced energy barrier can effectively constrain the migration of nanoparticles and thus prevent them from merging at a high temperature. We begin our MD studies with a control simulation (Fig. 5b), on defect-free graphene, two $3 \mathrm{~nm} \mathrm{Al} \mathrm{nanoparticles} \mathrm{are}$ initially located $5 \mathrm{~nm}$ apart from each other. Owing to thermal fluctuations at $2,200 \mathrm{~K}$, the $\mathrm{Al}$ nanoparticles will experience Brownian random walk and, when close enough to experience the weak van der Waals forces, eventually aggregate and coalesce to minimize the surface energy (Supplementary Movie 1). In contrast, if two $3 \mathrm{~nm} \mathrm{Al}$ nanoparticles are dispersed with the same distance on graphene with slit defects (Fig. 5c), the random walk of each nanoparticles is effectively confined within a domain demarcated by neighbouring defects, due to the barrier effect. As a result, the two nanoparticles remain dispersed without aggregation and coalescence (Supplementary Movie 2). Figure 5d further demonstrates that graphene boundaries can also serve as an effective barrier to confine the thermo-activated random walk of the nanoparticles and thus prevent the coalescence of neighbouring nanoparticles (Supplementary Movie 3). Furthermore, as the chemical reactivity of the carbon atoms at the defect sites is relatively higher than those away from defects, the chemical bonding between these carbon atoms and the nanoparticles could also possibly occur ${ }^{52}$, which could further anchor the nanoparticles and prevent them from merging at high temperature.

Al nanoparticles-RGO as switchable energetic material. Among their many potential applications, these well-dispersed nanoparticles without surface oxidation in a conductive matrix are particularly well suited for use as energetic materials, which is a class of materials defined by the ability to very rapidly convert stored chemical potential energy to thermal energy, to produce work (for example, propellants and explosives). There has been a recent drive to employ nanoparticles in such applications, because they have been demonstrated to burn faster and have lower ignition temperatures than their microsized counterparts ${ }^{53-56}$. However, reactive nanoparticles are also likely to agglomerate and to suffer rapid loss of surface area due to coalescence and sintering on heating, which diminishes the advantages of using nanoparticles (that is, enhanced specific surface area and shorter diffusion distances $)^{57-59}$. Native oxide layers are another issue as even the typical 2-5 nm layer that forms on contact with air can represent a significant portion $(\sim 30 \%)$ of a nanoparticle's mass ${ }^{53}$. In addition, one major practical concern for nanoenergetic materials is safety, particularly in regards to avoiding unplanned ignition event. Thus, an ideal energetic system would stabilize the nanostructure against agglomeration and oxidation so the full potential of the nanoscale could be realized, while being insensitive to accidental ignition. Here we demonstrate that the nAl-RGO produced in this study achieves much of the ideality outlined above.

The energetic nature of this material is illustrated in Fig. 6. The first line of images in Fig. 6a shows frames from high-speed video of activated 1:1 nAl-RGO that was flooded with $\mathrm{O}_{2}$ to produce a violent reaction. The sample $(1,700 \mu \mathrm{m}$ long, $410 \mu \mathrm{m}$ wide and 
a

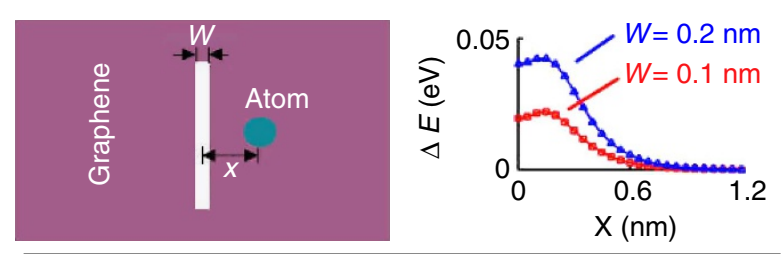

b

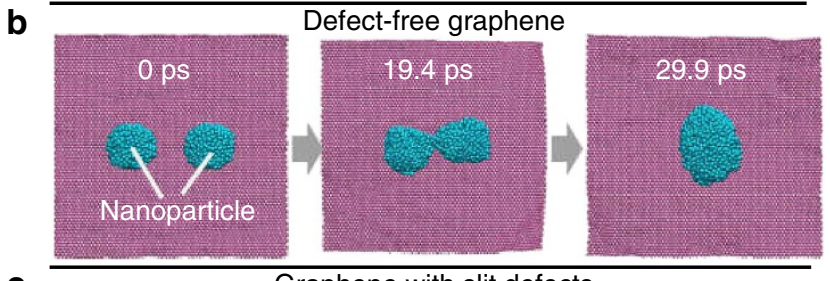

c

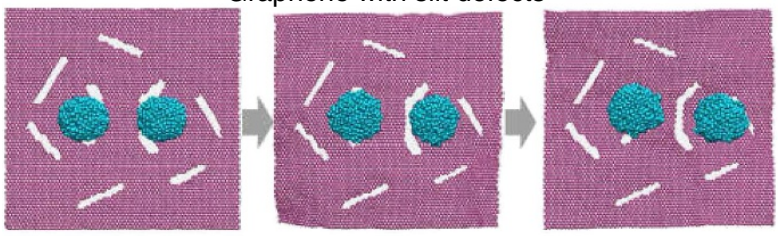

d

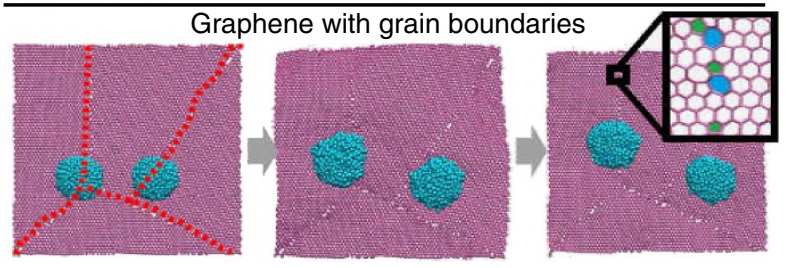

Figure 5 | Barrier effect of defects on structural stability of

nanoparticles. (a) Schematic of an atom located with a distance of $x$ to the center of a slit defect of width $w$ in graphene and the evolution of potential energy as the function of $x$, clearly showing an energy barrier as the atom approaches the defect. (b) On defect-free graphene, two neighbouring 3-nm Al nanoparticles are initially apart from each other by $5 \mathrm{~nm}$, then migrate by Brownian random walk at 2,200 K and eventually aggregate and coalesce into a single particle, driven by surface energy minimization.

(c) On graphene with slit defects, two $3 \mathrm{~nm}$ Al nanoparticles with the same initial dispersion distance are shown to be effectively confined within the domain demarcated by the slit defects, owing to the barrier effect. The two nanoparticles remain dispersed without coalescence at 2,200 K. (d) On graphene with grain boundaries (as outlined by dotted lines in left panel, mainly made of pentagon-heptagon paired defects), the two nanoparticles also remain dispersed without coalescence at 2,200 K.

$4 \mu \mathrm{m}$ thick) was connected to electrical leads with silver paste and heating was achieved by passing current $(\sim 70 \mathrm{~mA})$ through the material. Before reaction, this sample was held at a temperature ( $\sim 1,500 \mathrm{~K}$ based on previous pyrometry results) for $2 \mathrm{~min}$ in a chamber pumped to $0.001 \mathrm{~atm}$, to activate it by allowing for reduction of extraneous $\mathrm{Al}_{2} \mathrm{O}_{3}$ on the outer surface of RGO (see discussion below). Following activation, the chamber was then opened to 3 atm of pure $\mathrm{O}_{2}$, while the material was still hot. The second row of images in Fig. 6a shows the reaction of RGO alone under the same conditions, which was far less violent and thus illustrates the immense enhancement achieved by adding $\mathrm{Al}$ nanoparticles to the system.

Reactivity is quantified in Fig. $6 \mathrm{~b}$ by plotting the temporal emission intensity taken from the individual frames of high-speed video (frames from the specific videos used to make this plot are shown in Supplementary Fig. 12). The plot shows that RGO alone, both with activation (preheated in vacuum; black line) and without activation (heated directly in 3 atm of $\mathrm{O}_{2}$; green line) exhibited similar weak emission. The nAl-RGO film without activation (blue line) shows modest enhanced emission over
RGO, indicating the presence of some active $\mathrm{Al}$ nanoparticles. By contrast, the activated film material (that is, material that was thermally processed before admission of the oxidizer; red line) shows a greatly enhanced emission event, consistent with the differences seen in Fig. 6a. It is noteworthy that although different heating and oxygen introduction conditions were used for the 'with activation' and the 'without activation' samples, the similar performance of the control RGO enables this comparison between the nAl-RGO experiments under different conditions.

Our hypothesis for this observed behaviour is that after the initial synthesis of the nanoparticles, when the film is exposed to air, the $\mathrm{Al}$ nanoparticles on the outer surface of the RGO are oxidized, which diminishes the reactivity when the sample is heated directly without activation. During activation, the oxide is reduced by the high temperature and the presence of carbon during activation, which leaves a highly reactive nanofuel that can fully participate in the reaction. Therefore, the RGO matrix provides both an avenue for overcoming nanoparticle oxidation and physical stabilization against aggregation and coalescence. More information on this carbothermic reduction process can be found in Supplementary Fig. 13 and Supplementary Note 4.

In regards to safety, the difference between the two composite cases is evidence that energetic release of this material can be tuned in situ in a manner analogous to an ON-OFF switch. Such a capability would greatly enhance the handling of energetic materials and enable the use of materials that would be nominally too dangerous to employ. This concept is further supported by the failure of the non-activated material to burn when heated with a butane torch as shown in Supplementary Fig. 14 and Supplementary Note 5. Another benefit of material is that it can be processed with $3 \mathrm{D}$ printing technique. Applications such as micropyrotechnics and micropropulsion are dependent on patterning energetic material with fine control, which is something printing provides ${ }^{60}$. An example of this is shown in Fig. 6c, which demonstrates printing by a $3 \mathrm{D}$ printer using liquid $\mathrm{GO}-\mathrm{Al}$ ink $^{61-63}$. In this case, the in situ nanoparticle generation and activation can be done directly by Joule heating of the printed material as demonstrated by the emission in the far right image in Fig. 6c. Experimental details are described in Supplementary Figs 15,16 and Supplementary Note 6 .

\section{Discussion}

We report fine nanoparticles with an average size of $10 \mathrm{~nm}$ can be formed in situ inside a conductive RGO matrix at high temperature. The method is particularly attractive for reactive or energetic nanoparticles. For example, surface-oxidation-free $\mathrm{Al}$ nanoparticles are uniformly distributed without any agglomeration. This in situ methodology by Joule heating for fast nanoparticle fabrication avoids two typical challenges in active nanoparticle synthesis and assembly, which are surface oxidation and agglomeration due to the large specific surface area. Defect-guided self-assembly is likely to be the underlying mechanism of the superior structural stability of the resulting nanoparticles, supported by MD simulations. Other features that are unique for this in situ formation process of nanoparticles in functional structure include the following: (1) synthesis process can be completed within $\sim 10 \mathrm{~ms}$; (2) the nanoparticles are small with a size around $10 \mathrm{~nm}$; (3) the layered RGO as a barrier can protect the nanoparticles from oxidation; (4) the method can be applicable to a wide range of materials as demonstrated for $\mathrm{Sn}, \mathrm{Si}$, $\mathrm{Au}$ and Pd. The nAl-RGO composites were demonstrated to have direct applicability as an energetic material with a unique property of switchable reactivity. The RGO matrix was found to enable both initiation of the composites and reduction of extraneous oxide caused by exposure to air. Highly dispersed 
a
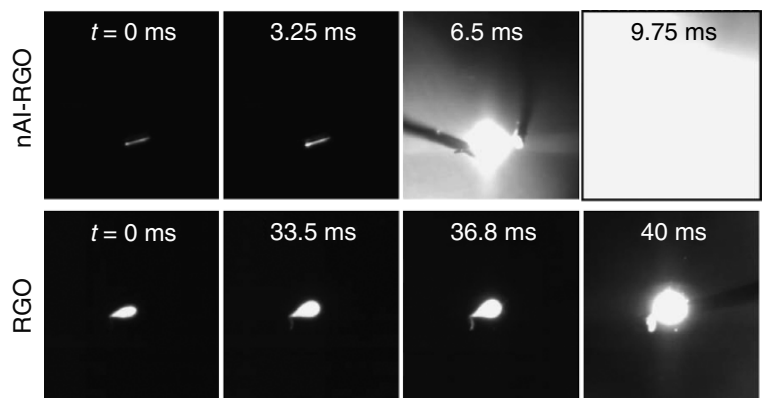

C
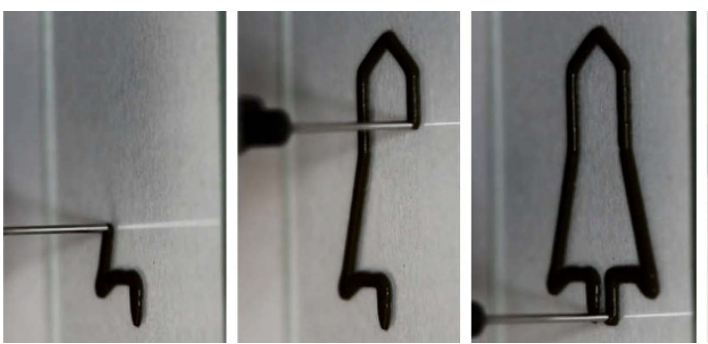

b
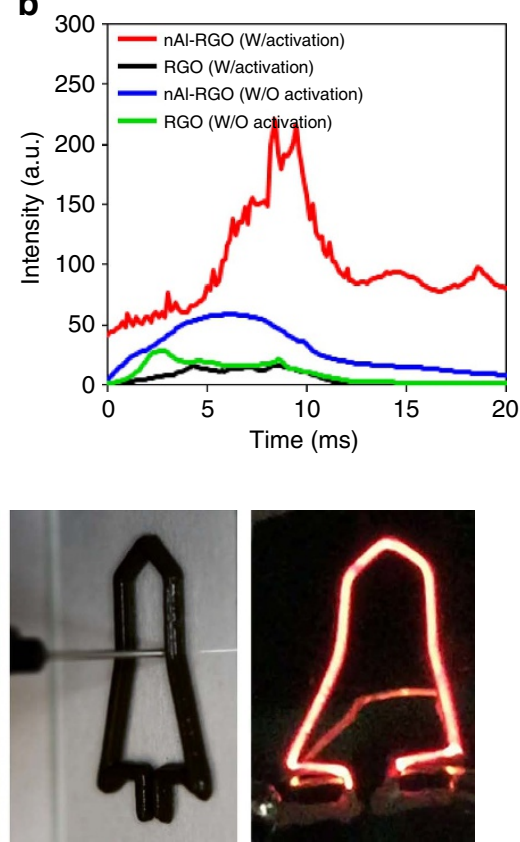

Figure 6 | Application of Al nanoparticles in RGO network as an energetic material. (a) Frames from high-speed videos with $142 \mu$ s exposure time of activated nAl-RGO and RGO that werekept hot and flooded with $3 \mathrm{~atm} \mathrm{O}_{2}$. Time 0 for both materials was arbitrarily defined and the last frame shown is the one with peak integrated intensity. (b) The normalized integrated intensity taken from the video frames ( $20 \mu \mathrm{s}$ exposure) of $\mathrm{nAl-RGO}$ and RGO samples that were activated (as in a) and that were subject to direct heating in 3 atm of $\mathrm{O}_{2}$ without activation. (c) Thee-dimensional printing process of Al-RGO and in situ formation to nAI-RGO by fast Joule heating.

and oxidation-free, agglomeration-free nanoparticles embedded in a highly conductive matrix can also be used for batteries, catalysts and many other emerging applications.

\section{Methods}

Materials synthesis. Detailed fabrication processes of various nanoparticles-RGO films are included in the Supplementary Methods.

Materials characterization. The morphology of the nanoparticles-RGO network was examined by a Hitachi SU-70 field-emission SEM. The SEM images were recorded in secondary electron mode. The samples for SEM surface characterization were prepared directly by adhering the freestanding film to the carbon tape on the stage. The as-prepared freestanding film was broken by tweezers or peeled off by double-sided cellotape for revealing internal surface morphology. TEM JEOL JEM $2100 \mathrm{~F}$ performed at an accelerating voltage of $200 \mathrm{kV}$ was also employed for the nanoparticle-RGO network morphology characterization. The TEM images were recorded in both high-resolution mode and bright-field mode. For preparing the samples for TEM characterization, the as-prepared nAl-RGO film was added in $1 \mathrm{ml}$ of ethanol followed by sonication for $\sim 10 \mathrm{~h}$ at room temperature until a suspension was achieved. Next, a suspension droplet was applied onto the copper grid. Ten samples for each type of nanoparticles in RGO were fabricated for particles size distribution. Particle size distribution was acquired by statistically analysing a large number of SEM and TEM images using ImageJ software. Raman spectroscopy was performed with a commercial micro Raman spectrometer (Labram Aramis model manufactured by Horiba Jobin Yvon) using a $633 \mathrm{~nm} \mathrm{He}$ Ne laser. The thickness of GO nanosheets was determined by atomic force microscopy. Temperature characterizations are included in Supplementary Methods.

Modelling. We use the large-scale atomic/molecular massively parallel simulator ${ }^{64}$ to perform MD simulations. The non-bonded interaction between carbon atoms in the graphene and the $\mathrm{Al}$ atoms in the catalyst nanoparticle is modelled by the Lennard-Jones 12-6 potential $\left(V(r)=4 \epsilon\left(\frac{\sigma^{12}}{r^{12}}-\frac{\sigma^{6}}{r^{6}}\right)\right)$ with the parameters $\epsilon=\epsilon_{A l-C}=0.0079 \mathrm{eV}$ and $\sigma=\sigma_{A l-C}=0.39495 \mathrm{~nm}$, as developed through the customary Lorentz-Berthelot mixing rules, using $\mathrm{Al}-\mathrm{Al}$ and $\mathrm{C}-\mathrm{C}$ parameters from the Universal Force Field ${ }^{65}$. We use the embedded atom potential file with the large-scale atomic/molecular massively parallel simulator distribution to describe the bounded interaction between $\mathrm{Al}$ atoms in the nanoparticle. The simulation is performed on a canonical ensemble, controlled by a Nosé-Hoover thermostat at $2,200 \mathrm{~K}$. The time step is $0.0001 \mathrm{ps}$. In all simulations, the Al particle is initially modelled $3 \mathrm{~nm}$ in diameter and $5 \mathrm{~nm}$ in the distance between centres. The graphene sheet is $15 \mathrm{~nm} \times 15 \mathrm{~nm}$ in size.

Energetic characterization. The reactivity of the samples was characterized using a high-speed camera (Phantom v12.1) and a chamber that could be evacuated to $0.001 \mathrm{~atm}$ and maintain a pressure up to $3 \mathrm{~atm}$. The material was connected to electrical leads using silver paste, inserted into the chamber and was Joule heated with $\sim 70 \mathrm{~mA}$ for activation and to drive reaction. Pure $\mathrm{O}_{2}$ gas at 3 atm was used as an oxidizer. For the 'with activation' samples, the material was heated first in vacuum and oxidizer was flowed into the chamber by opening a valve to a pressurized line, while the material was held at high temperature. For the 'without activation' samples, the chamber was pressurized before heating. It is noteworthy that particular care was taken to keep the sample width, thickness and length constant throughout all experiments to ensure consistent heating conditions.

Data availability. The data that support the findings of this study are available from the corresponding author upon request.

\section{References}

1. Atwater, H. A. \& Polman, A. Plasmonics for improved photovoltaic devices. Nat. Mater. 9, 205-213 (2010).

2. Maier, S. A. et al. Local detection of electromagnetic energy transport below the diffraction limit in metal nanoparticle plasmon waveguides. Nat. Mater. 2, 229-232 (2003).

3. Zhu, G. et al. Toward large-scale energy harvesting by a nanoparticle-enhanced triboelectric nanogenerator. Nano Lett. 13, 847-853 (2013).

4. Wang, C. et al. Self-healing chemistry enables the stable operation of silicon microparticle anodes for high-energy lithium-ion batteries. Nat. Chem. 5, 1042-1048 (2013).

5. Wu, H. et al. Stable Li-ion battery anodes by in-situ polymerization of conducting hydrogel to conformally coat silicon nanoparticles. Nat. Commun. 4, 1943 (2013).

6. Magasinski, A. et al. High-performance lithium-ion anodes using a hierarchical bottom-up approach. Nat. Mater. 9, 353-358 (2010).

7. Prieto, G., Zečević, J., Friedrich, H., de Jong, K. P. \& de Jongh, P. E. Towards stable catalysts by controlling collective properties of supported metal nanoparticles. Nat. Mater. 12, 34-39 (2013).

8. Chen, X. et al. Synthesis of 'clean' and well-dispersive Pd nanoparticles with excellent electrocatalytic property on graphene oxide. J. Am. Chem. Soc. 133, 3693-3695 (2011). 
9. Peng, G. et al. Diagnosing lung cancer in exhaled breath using gold nanoparticles. Nat. Nanotechnol. 4, 669-673 (2009).

10. Liu, Y., Dong, X. \& Chen, P. Biological and chemical sensors based on graphene materials. Chem. Soc. Rev. 41, 2283-2307 (2012).

11. Lee, J.-H. et al. Artificially engineered magnetic nanoparticles for ultra-sensitive molecular imaging. Nat. Med. 13, 95-99 (2007).

12. Petros, R. A. \& DeSimone, J. M. Strategies in the design of nanoparticles for therapeutic applications. Nat. Rev. Drug Discov. 9, 615-627 (2010).

13. Ghorbani, H. A review of methods for synthesis of $\mathrm{Al}$ nanoparticles. Orient. J. Chem. 30, 1941-1949 (2014).

14. Tsuzuki, T. Commercial scale production of inorganic nanoparticles. Int. J. Nanotechnol. 6, 567 (2009).

15. Gauthier, M. et al. A low-cost and high performance ball-milled Si-based negative electrode for high-energy Li-ion batteries. Energy Environ. Sci. 6, 2145-2155 (2013)

16. Sheng, H. W., Lu, K. \& Ma, E. Melting and freezing behavior of embedded nanoparticles in ball-milled $\mathrm{Al}-10 \mathrm{wt} \% \mathrm{M}(\mathrm{M}=\mathrm{In}, \mathrm{Sn}, \mathrm{Bi}, \mathrm{Cd}, \mathrm{Pb})$ mixtures. Acta Mater. 46, 5195-5205 (1998).

17. Pronko, P. P., Zhang, Z. \& VanRompay, P. A. Critical density effects in femtosecond ablation plasmas and consequences for high intensity pulsed laser deposition. Appl. Surf. Sci. 208-209, 492-501 (2003).

18. Eliezer, S. et al. Synthesis of nanoparticles with femtosecond laser pulses. Phys. Rev. B 69, 144119 (2004).

19. Amoruso, S., Ausanio, G., Bruzzese, R., Vitiello, M. \& Wang, X. Femtosecond laser pulse irradiation of solid targets as a general route to nanoparticle formation in a vacuum. Phys. Rev. B 71, 033406 (2005).

20. Sindhu, T. K., Sarathi, R. \& Chakravarthy, S. R. Generation and characterization of nano aluminium powder obtained through wire explosion process. Bull. Mater. Sci. 30, 187-195 (2007).

21. Sindhu, T. K., Sarathi, R. \& Chakravarthy, S. R. Understanding nanoparticle formation by a wire explosion process through experimental and modelling studies. Nanotechnology 19, 025703 (2008).

22. Swihart, M. T. Vapor-phase synthesis of nanoparticles. Curr. Opin. Colloid Interface Sci. 8, 127-133 (2003)

23. Pandey, P. A. et al. Physical vapor deposition of metal nanoparticles on chemically modified graphene: observations on metal-graphene interactions. Small Weinh. Bergstr. Ger. 7, 3202-3210 (2011)

24. Chung, S.-W. et al. Top-down meets bottom-up: dip-pen nanolithography and DNA-directed assembly of nanoscale electrical circuits. Small 1, 64-69 (2005).

25. Wang, Y. \& Xia, Y. Bottom-up and top-down approaches to the synthesis of monodispersed spherical colloids of low melting-point metals. Nano Lett. 4, 2047-2050 (2004)

26. Habas, S. E., Lee, H., Radmilovic, V., Somorjai, G. A. \& Yang, P. Shaping binary metal nanocrystals through epitaxial seeded growth. Nat. Mater. 6, 692-697 (2007)

27. Henzie, J., Grünwald, M., Widmer-Cooper, A., Geissler, P. L. \& Yang, P. Self-assembly of uniform polyhedral silver nanocrystals into densest packings and exotic superlattices. Nat. Mater. 11, 131-137 (2012).

28. Sun, Y. \& Xia, Y. Shape-controlled synthesis of gold and silver nanoparticles. Science 298, 2176-2179 (2002)

29. Cho, E. C., Zhang, Q. \& Xia, Y. The effect of sedimentation and diffusion on cellular uptake of gold nanoparticles. Nat. Nanotechnol. 6, 385-391 (2011).

30. Sau, T. K. \& Murphy, C. J. Room temperature, high-yield synthesis of multiple shapes of gold nanoparticles in aqueous solution. J. Am. Chem. Soc. 126, 8648-8649 (2004)

31. Murphy, C. J. et al. Anisotropic metal nanoparticles: synthesis, assembly, and optical applications. J. Phys. Chem. B 109, 13857-13870 (2005).

32. $\mathrm{Hu}, \mathrm{M}$. et al. Gold nanostructures: engineering their plasmonic properties for biomedical applications. Chem. Soc. Rev. 35, 1084 (2006).

33. Xia, Y., Gates, B., Yin, Y. \& Lu, Y. Monodispersed colloidal spheres: old materials with new applications. Adv. Mater. 12, 693-713 (2000).

34. Maillard, F. et al. Influence of particle agglomeration on the catalytic activity of carbon-supported Pt nanoparticles in CO monolayer oxidation. Phys. Chem. Chem. Phys. 7, 385-393 (2005).

35. Nakamura, R., Tokozakura, D., Nakajima, H., Lee, J.-G. \& Mori, H. Hollow oxide formation by oxidation of $\mathrm{Al}$ and $\mathrm{Cu}$ nanoparticles. J. Appl. Phys. 101, 074303 (2007)

36. Gromov, A. A. \& Teipel, U. Metal Nanopowders: Production, Characterization, and Energetic Applications (John Wiley \& Sons, 2014).

37. Jin, Y. et al. Simultaneous purification and perforation of low-grade Si sources for lithium-ion battery anode. Nano Lett. 15, 7742-7747 (2015).

38. Su, X. et al. Silicon-based nanomaterials for lithium-ion batteries: a review. $A d v$. Energy Mater. 4, 1300882 (2014).

39. Su, Y. et al. Impermeable barrier films and protective coatings based on reduced graphene oxide. Nat. Commun. 5, 4843 (2014)

40. Berciaud, S. et al. Electron and optical phonon temperatures in electrically biased graphene. Phys. Rev. Lett. 104, 227401 (2010)
41. Yu, D. \& Dai, L. Voltage-induced incandescent light emission from large-area graphene films. Appl. Phys. Lett. 96, 143107 (2010).

42. Kim, Y. D. et al. Bright visible light emission from graphene. Nat. Nanotechnol. 10, 676-681 (2015).

43. Dorgan, V. E., Behnam, A., Conley, H. J., Bolotin, K. I. \& Pop, E. High-field electrical and thermal transport in suspended graphene. Nano Lett. 13, 4581-4586 (2013).

44. Kim, K. et al. High-temperature stability of suspended single-layer graphene. Phys. Status Solid. Rapid Res. Lett. 4, 302-304 (2010).

45. Cho, E. S. et al. Graphene oxide/metal nanocrystal multilaminates as the atomic limit for safe and selective hydrogen storage. Nat. Commun. 7, 10804 (2016).

46. Menzel, R. et al. Joule heating characteristics of emulsion-templated graphene aerogels. Adv. Funct. Mater. 25, 28-35 (2015).

47. Yavari, F. et al. High sensitivity gas detection using a macroscopic three-dimensional graphene foam network. Sci. Rep. 1, 166 (2011).

48. Hu, H., Zhao, Z., Zhou, Q., Gogotsi, Y. \& Qiu, J. The role of microwave absorption on formation of graphene from graphite oxide. Carbon 50, 3267-3273 (2012).

49. Ferrari, A. C. \& Basko, D. M. Raman spectroscopy as a versatile tool for studying the properties of graphene. Nat. Nanotechnol. 8, 235-246 (2013).

50. Ghosh, T. et al. Solution-processed graphite membrane from reassembled graphene oxide. Chem. Mater. 24, 594-599 (2012).

51. Huang, Y., Zhu, S. \& Li, T. Line defects guided molecular patterning on graphene. Appl. Phys. Lett. 104, 093102 (2014).

52. Kim, K. et al. Selective metal deposition at graphene line defects by atomic layer deposition. Nat. Commun. 5, 4781 (2014).

53. Dreizin, E. L. Metal-based reactive nanomaterials. Prog. Energy Combust. Sci. 35, 141-167 (2009)

54. Yetter, R. A., Risha, G. A. \& Son, S. F. Metal particle combustion and nanotechnology. Proc. Combust. Inst. 32, 1819-1838 (2009).

55. Sullivan, K. T., Kuntz, J. D. \& Gash, A. E. The role of fuel particle size on flame propagation velocity in thermites with a nanoscale oxidizer. Propellants Explos. Pyrotech. 39, 407-415 (2014)

56. Huang, Y., Risha, G. A., Yang, V. \& Yetter, R. A. Combustion of bimodal nano/micron-sized aluminum particle dust in air. Proc. Combust. Inst. 31, 2001-2009 (2007).

57. Chakraborty, P. \& Zachariah, M. R. Do nanoenergetic particles remain nano-sized during combustion? Combust. Flame 161, 1408-1416 (2014).

58. Egan, G. C., Sullivan, K. T., LaGrange, T., Reed, B. W. \& Zachariah, M. R. In situ imaging of ultra-fast loss of nanostructure in nanoparticle aggregates. J. Appl. Phys. 115, 084903 (2014).

59. Zong, Y., Jacob, R. J., Li, S. \& Zachariah, M. R. Size resolved high temperature oxidation kinetics of nano-sized titanium and zirconium particles. J. Phys. Chem. A 119, 6171-6178 (2015).

60. Rossi, C. \& Estève, D. Micropyrotechnics, a new technology for making energetic microsystems: review and prospective. Sens. Actuators Phys. 120, 297-310 (2005).

61. Kim, J. H. et al. 3D printing of reduced graphene oxide nanowires. Adv. Mater. 27, 157-161 (2015)

62. García-Tuñon, E. et al. Printing in three dimensions with graphene. Adv. Mater. 27, 1688-1693 (2015).

63. Fu, K. et al. Graphene oxide-based electrode inks for 3D-printed lithium-ion batteries. Adv. Mater. 28, 2587-2594 (2016).

64. Plimpton, S. Fast parallel algorithms for short-range molecular dynamics. J. Comput. Phys. 117, 1-19 (1995).

65. Rappe, A. K., Casewit, C. J., Colwell, K. S., Goddard, W. A. \& Skiff, W. M. UFF, a full periodic table force field for molecular mechanics and molecular dynamics simulations. J. Am. Chem. Soc. 114, 10024-10035 (1992).

\section{Acknowledgements}

Dr L.H. acknowledges the financial support from the dean's office for the equipmen setup. We acknowledge the support of the Maryland NanoCenter and its AIMLab. Y.C. acknowledges the China Scholarship Council (CSC) for financial support. The work of G.C.E. and M.R.Z. was supported through an Office of Naval Research (ONR) Multidisciplinary University Research Initiative (MURI) programme.

\section{Author contributions}

Y.C., J.W. and L.H. conceived the idea of fast nanoparticle formation and designed the experiment. G.C.E. and M.R.Z. demonstrated the energetic capabilities. S.Z. and T.L. carried out the modelling, to understand the nanoparticle formation mechanism. All authors contributed to the manuscript writing.

\section{Additional information}

Supplementary Information accompanies this paper at http://www.nature.com/ naturecommunications 
Competing financial interests: The authors declare no competing financial interests.

Reprints and permission information is available online at http://npg.nature.com/ reprintsandpermissions/

How to cite this article: Chen, Y. et al. Ultra-fast self-assembly and stabilization of reactive nanoparticles in reduced graphene oxide films. Nat. Commun. 7:12332 doi: $10.1038 /$ ncomms12332 (2016). (c) (i) This work is licensed under a Creative Commons Attribution 4.0 International License. The images or other third party material in this article are included in the article's Creative Commons license, unless indicated otherwise in the credit line; if the material is not included under the Creative Commons license, users will need to obtain permission from the license holder to reproduce the material. To view a copy of this license, visit http://creativecommons.org/licenses/by/4.0/

(C) The Author(s) 2016 